\title{
Null Surfaces and the Bach Equations
}

\author{
Mirta Iriondo, Carlos N. Kozameh, Alejandra Rojas \\ FaMAF , Universidad Nacional de Córdoba, 5000 Córdoba , Argentina
}

\begin{abstract}
It is shown that the integrability conditions that arise in the Null Surface Formulation (NSF) of general relativity (GR) impose a field equation on the local null surfaces which is equivalent to the vanishing of the Bach tensor. This field equation is written explicitly to second order in a perturbation expansion.

The field equation is further simplified if asymptotic flatness is imposed on the underlying space-time. The resulting equation determines the global null surfaces of asymptotically flat, radiative space-times. It is also shown that the source term of this equation is constructed from the free Bondi data at $\mathcal{I}$.

Possible generalizations of this field equation are analyzed. In particular we include other field equations for surfaces that have already appeared in the literature which coincide with ours at a linear level. We find that the other equations do not yield null surfaces for GR.
\end{abstract}

\section{INTRODUCTION}

Recently, a new formulation of GR has been developed where, instead of a metric $g_{a b}(x)$ on the space-time, the basic variables are two functions defined on the bundle of null directions. Denoting by $x^{a}$ the points of the space-time, by $(\zeta, \bar{\zeta})$ the parameters on the sphere of null directions, the basic variables in the so called Null Surface Formulation (NSF) of GR are:

- a function $Z\left(x^{a}, \zeta, \bar{\zeta}\right)$ such that, for fixed values of $(\zeta, \bar{\zeta})$, its level surfaces, i.e. $Z=$ const. are characteristic surfaces on the space-time,

- another scalar function $\Omega$ that, for fixed $(\zeta, \bar{\zeta})$, plays the role the conformal factor, i.e., fixes the scale to measure distances on the space-time.

It follows from the above properties that $Z$ yields the conformal structure of the spacetime and thus determines nine out of the ten components of the metric $g_{a b}(x)$ whereas $\Omega$ fixes the last component. The scalar $\Omega$, however, plays a more important role in the dynamics of the theory.

The variables $Z$ and $\Omega$ satisfy three differential equations with the following geometrical interpretation:

1. A complex first order differential equation for $\Omega$ and $Z$ given schematically as

$$
ð \Omega=W[Z] \Omega
$$


with $\partial$ (essentially $\frac{\partial}{\partial \zeta}$ ) the covariant derivative on the sphere of null directions [1], [2] and $W$ a functional that only depends on $Z$

2. A complex differential equation for $Z$ involving derivatives with respect to $\left(x^{a}, \zeta, \bar{\zeta}\right)$.

3. A real equation for $\Omega$ and $Z$ that adopts the form

$$
\partial_{1}^{2} \Omega=Q[Z] \Omega
$$

with $\partial_{1}$ a derivative along a null geodesic on the $Z=$ const. hypersurface and $Q$ another functional that only depends on $Z$.

The first two equations must be satisfied by $Z$ and $\Omega$ if they are to yield a conformal metric for the space-time. These equations, called metricity conditions $\left(m_{I}\right)$ and $\left(m_{I I}\right)$ are purely kinematical, since they are valid for any (conformal) metric with Lorentzian signature. The last equation, denoted by (E) is equivalent to the vacuum Einstein equations for a lorentzian metric.

If we interpret $(\mathrm{E})$ as an equation for $\Omega$, then the full dynamics can be thought as an ODE for a single scalar, rather than the usual ten equations for a metric tensor. The reader should be reminded, however, that the cut function $Z$ that enters in (E) cannot be arbitrary since it must satisfy the metricity conditions. In fact, the three equations $(\mathrm{mI}),(\mathrm{mII})$, and (E) must be solved simultaneously for $\Omega$ and $Z$ to obtain a consistent solution to the dynamical problem.

In this paper we reexamine the field equations of the NSF and show that the dynamics of the conformal structure can be written as a single equation for the function $Z$.

We first observe that the scalar $\Omega$ satisfies two differential equations, $(\mathrm{E})$ and $\left(m_{I}\right)$, where two functionals of $Z, Q$ and $W$, play the role of source terms. Thus, for a non-trivial solution to exist integrability conditions must be imposed on $Q$ and $W$.

We then study the integrability conditions of those equations and show that they impose a differential equation on the local null surfaces of the NSF. The resulting equation, together with the metricity condition $\left(m_{I I}\right)$, turns out to be equivalent to the the Bach equations, for a conformal metric and nicely tie this formalism with previous known results.

We also observe that the new equation, that will also be called Bach equation, and $\left(m_{I I}\right)$ are equations only for $Z$, i.e., they do not include the conformal factor. Thus, they describe the dynamics of the conformal structure of the space-time.

By imposing globality conditions on the Bach equation, we obtain an integrodifferential equation that determines the null surfaces of asymptotically flat, radiative space-times. In this equation the Bondi free data plays the role of a source term and its solutions have a dual meaning. They represent past null cones from points at $\mathcal{I}$ and 2 -surfaces at $\mathcal{I}$ representing the intersection of null cones from interior points with the null boundary. These 2-surfaces are called light cone cuts of null infinity or simply l.c. cuts.

Since the field equations that determine the l.c. cuts are so complicated one is tempted to ask if it is possible to generalize our formalism, keeping the kinematical arena provided by $\mathcal{I}$ but replacing the Bach equation with a simpler equation for the 2-surfaces. It is clear that, the surfaces satisfying the new equations would not yield conformal vacuum metrics but if they, on the other hand, satisfy $\left(m_{I I}\right)$ they would be characteristic surfaces of an underlying 
metric of the space-time with a given dynamical evolution due to an effective stress-energy tensor. In particular, we examine two possible generalizations of the field equations for the cuts and show that their solutions are not null surfaces of any metric since equation $\left(m_{I I}\right)$ is not satisfied. The lesson being learned here is that although the metricity conditions are kinematical in nature they play very important role when trying to describe GR via these non local variables.

This paper is structured as follows. A brief review of the NSF is presented in Section II whereas the "standard" derivation of the Bach equations is given in Section III. The integrability conditions of the NSF equations and a second order perturbation procedure together with the field equations for the light cone cuts are presented in Section IV. Possible generalizations of the light cone equations are given in Section V. All these alternative models fail to satisfy the metricity conditions. The main results are summarized and possible applications are discussed in the Conclusions. Several auxiliary calculations are presented in the Appendices.

\section{A BRIEF REVIEW OF NSF}

Since a thorough description of the NSF is already given in the literature [3], [4], [5], we will summarize its main results without any derivations and defer the inquisitive reader to the references.

The formalism first introduces a function $Z\left(x^{a}, \zeta, \bar{\zeta}\right)$ with $x^{a}$ points on the space-time and $(\zeta, \bar{\zeta})$ parameters on the sphere such that, for each $(\zeta, \bar{\zeta}), Z=$ const yields a family of surfaces on the manifold. We ask if $Z=$ const can be thought of null surfaces of a conformal metric. In general the answer is no since the equation :

$$
\hat{g}^{a b}(x) Z_{, a}\left(x^{c}, \zeta, \bar{\zeta}\right) Z_{, b}\left(x^{c}, \zeta, \bar{\zeta}\right)=0
$$

is an infinite set of algebraic equations, one for each value of $(\zeta, \bar{\zeta})$, for the nine coefficients of the conformal metric $\hat{g}^{a b}$. Therefore, we ask what are the conditions to be imposed on $Z$ such that a nontrivial $\hat{g}^{a b}(x)$ with Lorentzian signature exists. Two types of equations arise in the search for these conditions; the first type yields the components of the conformal metric, while the second type identifies necessary conditions to be satisfied by $Z$ (i.e the metricity conditions $\left(m_{I}\right)$ and $\left.\left(m_{I I}\right)\right)$. The conditions and metric components can be better expressed if we introduce a null coordinate system given by a set of 4-scalars associated with $Z$, namely

$$
\theta^{i}\left(x^{a}, \zeta, \bar{\zeta}\right)=\left(\theta^{0}, \theta^{+}, \theta^{-}, \theta^{1}\right)=(Z, ð Z, \bar{\jmath} Z, \precsim \bar{\partial} Z)=(u, w, \bar{w}, R)
$$

We will refer to the $\theta^{i}$ as the intrinsic coordinate system associated with the parametrized families of characteristic surfaces, labeled by the parameters $(\zeta, \bar{\zeta})$.

Introducing its associated 1-form basis $\theta_{a}^{i}=\partial_{a} \theta^{i}$ and dual vectors basis $\theta_{i}^{a}$ such that $\theta_{i}^{a} \theta_{a}^{j}=\delta_{i}^{j}$, we find that the conditions can be written in terms of two scalars

$$
\Lambda \equiv \check{\partial}^{2} Z, \quad \quad \Omega^{2} \equiv g^{a b} Z_{, a} \precsim \bar{\partial} Z_{, b},
$$

as: 


$$
\begin{aligned}
\left(m_{I}\right) \quad \precsim \Omega & =\frac{1}{2} W \Omega \\
\left(m_{I I}\right) \quad \partial_{-} \Lambda & =\frac{1}{2} \precsim \partial_{1} \Lambda-(W+ð(\ln q)) \partial_{1} \Lambda
\end{aligned}
$$

with $q=\left(1-\partial_{1} \Lambda \partial_{1} \bar{\Lambda}\right), \partial_{i} \equiv \frac{\partial}{\partial \theta^{2}}$, and

$$
W\left(1-\frac{1}{4} \partial_{1} \Lambda \partial_{1} \bar{\Lambda}\right)=\partial_{+} \Lambda+\frac{1}{2} \bar{\partial} \partial_{1} \Lambda-\frac{1}{2} ð(\ln q)-\frac{1}{4} \partial_{1} \Lambda \bar{\partial}(\ln q)+\frac{1}{2} \partial_{1} \Lambda\left(\bar{\Lambda}_{,-}+\frac{1}{2} ð \bar{\partial}_{1} \Lambda\right) .
$$

In the above equations, one is implicitly assuming that the scalars $\Lambda$ and $\Omega$ are functions of $(u, w, \bar{w}, R, \zeta, \bar{\zeta})$, i.e., one assumes that $\theta^{i}$ is a well behaved coordinate system, that the relationship (2) can be inverted to write $x^{a}$ as a function of $\theta^{i}$, and that the $x^{a}$ so obtained has been inserted in the r.h.s. of the defining equations for $\Lambda$ and $\Omega$.

It can also be shown that as a consequence of the metricity conditions $\left(m_{I}\right)$ and $\left(m_{I I}\right)$ one constructs a metric $\hat{g}^{a b}(x)$, i.e., independent of the parameters $(\zeta, \bar{\zeta})$, as a functional of $\Lambda$ and $\Omega$, (c.f. [4]). The explicit form of the metric is given as :

$$
\hat{g}^{a b}=\hat{g}^{i j}(\Lambda, \bar{\Lambda}) \theta_{i}^{a} \theta_{j}^{b}
$$

where $\hat{g}^{i j}=\Omega^{2} g^{i j}$ and

$$
\left(g^{i j}\right)=\left(\begin{array}{cccc}
0 & 0 & 0 & 1 \\
0 & -\partial_{1} \Lambda & -1 & g^{1+} \\
0 & -1 & -\partial_{1} \bar{\Lambda} & g^{1-} \\
1 & g^{1+} & g^{1-} & g^{11}
\end{array}\right)
$$

with $g^{1+}=-\frac{1}{2}\left(\bar{\partial} \partial_{1} \Lambda+\partial_{1} \Lambda \bar{W}\right)=\overline{g^{1-}}$ and $g^{11}=-2-\frac{1}{2} \bar{\partial}^{2} \partial_{1} \Lambda+ð \partial_{-} \bar{\Lambda}+\mathcal{O}\left(\Lambda^{2}\right)$.

So far the description of null surface theory has been completely kinematical. Our variables $(\Omega$ and $\Lambda)$ must satisfy the metricity conditions to define a Lorentzian metric. We now address the problem of finding a metric that in addition satisfies the trace free vacuum Einstein equations, i.e. $\hat{g}_{a b}$ satisfies

$$
\hat{R}_{a b}-\frac{1}{4} \hat{R} \hat{g}_{a b}=0
$$

Contracting this equation with $\theta_{1}^{a} \theta_{1}^{b}$ and using the explicit form of the metric in this coordinate system yields:

$$
\text { (E) } \quad \partial_{1}^{2} \Omega=Q \Omega
$$

where

$$
Q=-\frac{1}{q} \partial_{1}^{2} \bar{\Lambda} \partial_{1}^{2} \Lambda-\frac{3}{8 q^{2}}\left(\partial_{1} q\right)^{2}+\frac{1}{4 q} \partial_{1}^{2} q .
$$

Thus this equation together with the metricity conditions $\left(m_{I}\right)$ and $\left(m_{I I}\right)$ build up a system of differential equations equivalent to the vacuum Einstein equations.

It should be mentioned that the $\widetilde{\partial}$ operator used above adopts a different form when written in the $\theta^{i}$ coordinate system. We recall that when applied on a function $f\left(x^{a}, \zeta, \bar{\zeta}\right)$, $\partial$ is the covariant derivative with respect to $\zeta$. However, if we write $x^{a}=x^{a}\left(\theta^{i}, \zeta, \bar{\zeta}\right)$, then the operator adopts the form 


$$
\check{\partial}=\check{\partial}^{\prime}+w \partial_{0}+\Lambda \partial_{+}+R \partial_{-}+(\bar{\partial} \Lambda-2 w) \partial_{1},
$$

where $\widetilde{\partial}^{\prime}$ is the partial covariant derivative, i.e. keeping the $\theta^{i}$ fixed, and $\partial_{i} \equiv \frac{\partial}{\partial \theta^{i}}$.

It follows from this equation that the directional derivatives $\partial_{i}$ do not commute with the $\partial$ or $\bar{\partial}$ operators. We now write the commutation relations (and corresponding notation) between these derivatives since they will be used throughout this work.

Using (5) and denoting by $\delta_{i}=\left[\partial_{i}, \widetilde{\partial}\right]$ and $\bar{\delta}_{i}=\left[\partial_{i}, \bar{\partial}\right]$ (c.f. 伍) we obtain

$$
\begin{aligned}
& \delta_{1}=\partial_{-}+\partial_{1} \Lambda \partial_{+}+f_{1} \partial_{1}, \\
& \delta_{-}=\partial_{-} \Lambda \partial_{+}+f_{-} \partial_{1}, \\
& \delta_{+}=\partial_{0}-\left(\frac{2}{q}-f_{+}\right) \partial_{1}+\partial_{+} \Lambda \partial_{+}, \\
& \delta_{0}=f_{0} \partial_{1}+\partial_{0} \Lambda \partial_{+},
\end{aligned}
$$

where

$$
\begin{aligned}
& f_{1}=\frac{1}{q}\left(\partial_{+} \Lambda+\bar{\partial} \partial_{1} \Lambda+\partial_{-} \Lambda \partial_{1} \bar{\Lambda}+\left(\partial_{1} \Lambda \partial_{+} \bar{\Lambda}+\partial \partial_{1} \bar{\Lambda}+\partial_{-} \bar{\Lambda}\right) \partial_{1} \Lambda\right) \\
& f_{+}=\frac{1}{q}\left(\bar{\partial} \partial_{+} \Lambda+\partial_{-} \Lambda \partial_{+} \bar{\Lambda}+\left(\partial_{+} \Lambda \partial_{+} \bar{\Lambda}+\partial \partial_{+} \bar{\Lambda}+\partial_{0} \bar{\Lambda}\right) \partial_{1} \Lambda\right) \\
& f_{-}=\frac{1}{q}\left(-2 \partial_{1} \Lambda+\partial_{0} \Lambda+\bar{\partial} \partial_{-} \Lambda+\partial_{-} \Lambda \partial_{-} \bar{\Lambda}+\left(\partial_{-} \Lambda \partial_{+} \bar{\Lambda}+\partial \partial_{-} \bar{\Lambda}\right) \partial_{1} \Lambda\right) \\
& f_{0}=\frac{1}{q}\left(\bar{\partial} \partial_{0} \Lambda+\partial_{-} \Lambda \partial_{0} \bar{\Lambda}+\left(\partial_{0} \Lambda \partial_{+} \bar{\Lambda}+\partial \partial_{0} \bar{\Lambda}\right) \partial_{1} \Lambda\right) .
\end{aligned}
$$

For later use we also compute

$$
\left[\partial_{1}^{n}, ð\right]=\sum_{i=0}^{n-1} \partial_{1}^{n-1-i}\left[\partial_{1}, ð\right] \partial_{1}^{i} \quad \text { for } \quad n \in \mathbf{N} .
$$

and

$$
\begin{aligned}
\eta_{1}: & =\left[\partial_{1}, \delta_{1}\right] \\
& =\partial_{1} f_{1} \partial_{1}+\partial_{1}^{2} \Lambda \partial_{+}, \\
\eta_{2}: & =\left[\partial_{1}, \eta_{1}\right] \\
& =\partial_{1}^{2} f_{1} \partial_{1}+\partial_{1}^{3} \Lambda \partial_{+} \\
\eta_{3}: & =\left[\partial_{1}, \eta_{2}\right] \\
& =\partial_{1}^{3} f_{1} \partial_{1}+\partial_{1}^{4} \Lambda \partial_{+} .
\end{aligned}
$$

\section{THE BACH EQUATION}

The Bach tensor [6]

$$
B_{a b} \equiv \nabla^{m} \nabla^{n} C_{m a b n}+\frac{1}{2} R^{m n} C_{m a b n},
$$


is a trace free, symmetric, two index tensor constructed from the metric of the space-time with a particular useful feature: under rescaling of the metric it transforms with a conformal weight. As we will see below, the vanishing of the Bach tensor (a conformally invariant statement) arises as a necessary condition for a space-time to be conformal to an Einstein space-time [7].

Consider the conformal transformation

$$
g_{a b}=\Omega^{2} \hat{g}_{a b} \quad \text { or equivalently } \quad \hat{g}^{a b}=\Omega^{2} g^{a b}
$$

and assume that $\hat{g}_{a b}$ satisfies the trace free vacuum field equations (4). In terms of $\Omega$ and $g_{a b}$ these equations read

$$
\nabla_{a} \nabla_{b} \Omega-\frac{1}{4} \Delta \Omega g_{a b}+\frac{1}{2} \Omega\left(R_{a b}-\frac{1}{4} R g_{a b}\right)=0 .
$$

We now ask what conditions should be imposed on $g_{a b}$ so that a solution of (12) exists. If we consider eq. (12) as a second order differential equation for $\Omega$ it is then clear that, for a non trivial solution to exist, integrability conditions must be imposed on the metric $g_{a b}$. Taking the curl of (12) and using the Bianchi identities yields

$$
\Omega \nabla^{d} C_{a b c d}-\nabla^{d} \Omega C_{a b c d}=0 .
$$

Equation (13) is a new condition imposed on $\Omega$. Thus, we have to consider a new system consisting of equations (12) and (13).

In order to find the integrability conditions of this system, we take $\nabla^{a}$ to equation (13)

$$
\nabla^{a} \Omega \nabla^{d} C_{a b c d}-\left(\nabla^{a} \nabla^{d} \Omega\right) C_{a b c d}+\Omega \nabla^{a} \nabla^{d} C_{a b c d}-\nabla^{d} \Omega \nabla^{a} C_{a b c d}=0 .
$$

On the other side, assuming that equation (13) is satisfied and using the symmetries of the Weyl tensor, we obtain

$$
\begin{aligned}
\Omega \nabla^{a} \Omega \nabla^{d} C_{a b c d} & =\nabla^{a} \Omega \nabla^{d} \Omega C_{a b c d} \\
& =\Omega \nabla^{d} \Omega \nabla^{a} C_{a b c d}
\end{aligned}
$$

this implies that

$$
\nabla^{a} \Omega \nabla^{d} C_{a b c d}=\nabla^{d} \Omega \nabla^{a} C_{a b c d}
$$

Hence, replacing the last expression in (14) and using (12), we have

$$
B_{b c} \equiv \nabla^{a} \nabla^{d} C_{a b c d}+\frac{1}{2} R^{a d} C_{a b c d}=0 .
$$

Thus, the vanishing of the Bach tensor is necessary condition for a metric to be conformally related to an Einstein metric.

A brief review of the above procedure shows that we have taken the following identity

$$
\nabla^{a}\left(\nabla_{[a}\left(\nabla_{b]} \nabla_{c} \Omega-\frac{1}{4} g_{b] c} \Delta \Omega\right)-\frac{1}{2} C_{a b c}{ }^{d} \nabla_{d} \Omega\right)=0
$$

and used the field equations (12) and (13) to get rid of first and second derivatives of $\Omega$ yielding 


$$
\Omega B_{b c}=0 .
$$

We will call the l.h.s. of eq. (15) the generating integrability condition of the conformal Einstein equations. In general, given a PDE for a field we define its generating integrability condition as the equation constructed with the minimum number of commutators such that when the field equations are used, all derivatives of the field disappear. In particular, substituting eq. (12) and (13) in eq. (15) yields the integrability condition (16).

It is worth mentioning that a second integrability condition arises if we take the curl of (13) [7]. It can be shown, however, that for asymptotically flat space-times the vanishing of the Bach tensor is both necessary and sufficient [8]. Since our main motivation is to study this class of space-times we will not explicitly state the second condition.

\section{THE INTEGRABILITY CONDITIONS}

As we said before, equations $(\mathrm{E}),\left(m_{I}\right)$ and $\left(m_{I I}\right)$ are the field equations of the NSF. Note that $\left(m_{I}\right)$ (a complex differential equation) and (E) (an ODE) are two equations that must

be satisfied by a real function $\Omega$. It is therefore natural to study the integrability conditions of the following system of equations:

$$
\left.\begin{array}{ll}
(\mathrm{E}) & \partial_{1}^{2} \Omega=Q \Omega, \\
\left(m_{I}\right) & \check{\partial} \Omega=\frac{1}{2} W \Omega, \\
\left(\bar{m}_{I}\right) & \overline{\mathrm{\partial}} \Omega=\frac{1}{2} \bar{W} \Omega .
\end{array}\right\}
$$

In the above equations the integrability condition between $\left(m_{I}\right)$ and $\left(\bar{m}_{I}\right)$ is trivially satisfied because $\bar{\partial}^{2} \Lambda=\check{\partial}^{2} \bar{\Lambda}$ (c.f. [3]), it remains to study the integrability condition between (E) and $\left(m_{I}\right)$ (and complex conjugate).

Although in principle the procedure to generate the integrabibility conditions of (17) is straightforward, in practice it becomes quite cumbersome. To illustrate our approach we first consider a linearized version of (17), namely

$$
\left.\begin{array}{ll}
\left(\mathrm{E}^{\prime}\right) & \partial_{1}^{2} \Omega=0 \\
\left(m_{I}\right) & \check{\partial} \Omega=\frac{1}{2} W_{1} \Omega, \\
\left(\bar{m}_{I}\right) & \overline{\bar{\partial}} \Omega=\frac{1}{2} \bar{W}_{1} \Omega,
\end{array}\right\}
$$

with $W_{1} \equiv \partial_{+} \Lambda+\frac{1}{2} \bar{\partial} \partial_{1} \Lambda$.

Note that we are not writting (17) perturbately, i.e, we are not assuming that $\Omega$ has a prescribed behavior in $\Lambda$. It is important to remark that if we make a perturbation expansion for both $\Omega$ and the coefficients we do not obtain the linearized integrability conditions of (17). Rather, $\Omega$ has no prescribed behaviour on $\Lambda$ and the coefficients of eq.(18) are the linearized version of the corresponding coefficients of (17). 
Proposition IV.1 The generating integrability condition of equations (18) is

$$
2\left(\partial_{1} ð+3 \partial_{-}\right)\left[\partial_{1}^{2}, \partial_{+}\right] \Omega=\frac{1}{4} \Omega \partial_{1}^{5} \bar{\partial}^{2} \Lambda=-3 \Omega B_{11},
$$

where $B_{11}=B_{a b} \theta_{1}^{a} \theta_{1}^{b}$. The first equality is obtained via the field equations (18) and the second equality is a straightforward calculation of the linearized Bach tensor in the $\theta^{i}$ coordinate system. Thus, the integrability condition of (18) is given by

$$
\partial_{1}^{5 \bar{\partial}^{2} \Lambda}=0
$$

\section{Proof:}

The proof is tedious but straightforward. It essentially consists of applying on $\Omega$ the commutators $\left[\partial_{i} \partial_{j}, \widetilde{\partial}\right],\left[\partial_{i} \partial_{j}, \bar{\jmath}\right]$, and $\left[\partial_{i} \partial_{j}, \partial_{k}\right]$, for $i=(0,+,-, 1)$, to generate an enlarged system of differential equations such that when taking a further commutator, the first and second derivatives of $\Omega$ disappear.

The details are given in Appendix A. $\square$

Remark IV.1 It is important to note that the term $\partial_{1}^{5} \bar{\varpi}^{2} \Lambda$ in the above proposition has been obtained via two independent calculations. On one hand it arises from the generating integrability condition of eq. (18). On the other hand, it comes from an explicit calculation of the Bach tensor using a linearized metric in the $\theta^{i}$ coordinate system. This is not a mere coincidence. As we will see below, we will show that the generating integrability condition of (17) is the Bach tensor.

As mentioned before, the calculations leading to the integrability condition of the system (17) are quite involved but follow the same steps as the calculations done at first order in $\Lambda$ (for proof see Appendix B).

Proposition IV.2 The generating integrability condition of equations (17) is

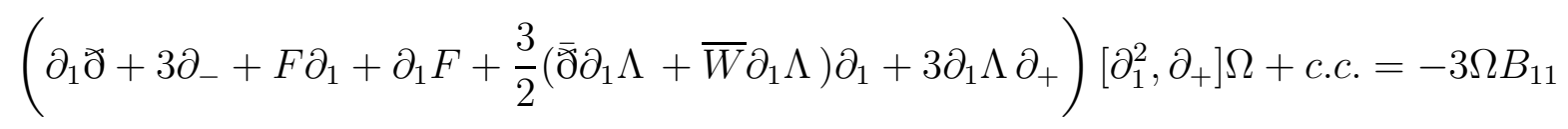

where c.c. means complex conjugate, and $F=F[\Lambda]$ is given in (B6).

Comparing the two propositions, we immediately see that extra terms of higher order in $\Lambda$ arise in Proposition IV.2. Those counterterms are needed to cancel the first derivatives of $\Omega$ obtained in the full calculation of the commutator $\left[\partial_{1}^{2}, \partial_{+}\right] \Omega$. 


\section{A. Second order perturbation}

Note that in Proposition IV.2 we have not calculated the explicit form of the integrability condition. Although in principle this can be done, this calculation is extremely involved and does not shed extra light on the subject. It is of interest however, to compute the condition up to second order in $\Lambda$ since it yields the first non trivial contribution to the field equations for our non local variables.

Consider the l.h.s. of equation $(\sqrt{19})$ and its complex conjugate. Since $\left[\partial_{1}^{2}, \partial_{+}\right] \Omega$ is $\mathcal{O}(\Lambda)$, we write $F$ up to order $\Lambda$ as $F=3 W_{1}$. Thus, to second order in $\Lambda$, equation (19) can be written as

$$
\left(\partial_{1} \partial+3 \delta_{1}+3 \partial_{1} W_{1}\right)\left[\partial_{1}^{2}, \partial_{+}\right] \Omega+\text { c.c. }=-3 \Omega B_{11}=0
$$

We thus have two independent methods to compute the integrability conditions to second order; computing the $[1,1]$ component of the Bach tensor to second order or using the l.h.s. of (20). It should be mentioned that blindly trying to compute the Bach tensor to second order in the $\theta^{i}$ coordinate system quickly exceeds the algebraic power of MAPLE (or alike). We have instead used the relationships that link several commutators of $\Omega$ with their corresponding tensorial counterparts to simplify the intermediate steps of the calculation. For example, using the relationships between $\left[\partial_{i} \partial_{j}, \partial\right] \Omega$ and $(12)$ and between $\left[\partial_{i} \partial_{j}, \partial_{k}\right] \Omega$ and (13) we can write the components of Christoffel symbols, Ricci, and Weyl tensor that are needed for this computation in terms of $Q$ and $W$. The detailed calculations are given in [9]. The final equation reads

$$
\begin{aligned}
\partial_{1}^{5 \varpi^{2}} \bar{\Lambda}= & \frac{1}{4} \partial_{1}^{4}\left(ð \partial_{1} \bar{\Lambda} W+\partial_{1} \bar{\Lambda} \partial W+\left[\delta_{1}, ð\right] \bar{\Lambda}\right)+3 \eta_{1} \partial_{1}^{2} \bar{W}+2 \eta_{2} \partial_{1} \bar{W}+\frac{1}{2} \eta_{3} \bar{W}+ \\
& +\frac{1}{2}\left(\partial_{1} \partial+3 \partial_{-}\right)\left(4 \partial_{+} Q+2 \partial_{1} \bar{\partial} Q+\frac{3}{2} \partial_{1}^{2} \bar{\Lambda} \partial_{1}^{2} W-\partial_{1} \bar{\Lambda} \partial_{1}^{3} W\right)+\frac{3}{2}\left(\frac{1}{2} \partial_{1} W \partial_{1}^{3} \bar{W}+\right. \\
& \left.-\frac{1}{2} \partial_{+} \partial_{1} \Lambda \partial_{1}^{3} \bar{W}+\partial_{1}^{2} \Lambda\left(\partial_{1}^{4} \bar{\Lambda}-\partial_{0} \partial_{1}^{3} \bar{\Lambda}+\frac{1}{2} \partial_{+}\left(3 \partial_{1}^{2} \bar{W}-2 \partial_{1}^{2} \bar{f}_{1}\right)\right)\right)+ \\
& -\frac{3}{4}\left(3 \partial_{1}^{2} \bar{W}-2 \partial_{1}^{2} \bar{f}_{1}\right) \partial_{1}^{2} W-\frac{3}{2} \partial_{1}^{3} \bar{\Lambda}\left(\frac{\partial \partial_{1}^{2} W}{2}-\partial_{-} \partial_{1} W\right)+\text { c.c. }
\end{aligned}
$$

For simplicity we have dropped the subindex 1 on $W$ in the r.h.s. of (21) since it can only contain linear terms in $\Lambda$. As we will see in the next subsection, the linearized $W$ vanishes for asymptotically flat space-times. This gives a considerable simplification in the resulting equation.

It is worth mentioning that using the generating integrability condition to second order yields the same result.

\section{B. Asymptotically Flat Spaces-Times}

As was shown by L. Mason, the vanishing of the Bach tensor is both a necessary and sufficient integrability condition for the existence of a solution to eq. (12) when the underlying space-time is asymptotically flat along null directions [8]. Thus, for those space-times eq. (21) yields the field equation for the non-local variable $Z$ up to second order in $\Lambda$. On 
the other hand, eq. (21) as it stands is valid even for metrics that are not asymptotically flat. It is thus clear that asymptotic flatness imposes extra conditions on $W$ and/or $Q$ on this equation. We now derive these conditions.

We first notice that $W$ on the r.h.s. must be known to first order in $\Lambda$ since it is always multiplied by factors of order $\Lambda$. To obtain the specific form of the linearized $W$ compatible with an asymptotic structure we should look for a pair $(\Omega, \Lambda)$ that satisfies eq. (18) with asymptotically flat boundary conditions.

Asymptotic flatness imposes the boundary condition $\lim _{R \rightarrow \infty} \Omega=1$ on the conformal factor. It then follows from the first equation (E') that $\Omega=1+\mathcal{O}\left(\Lambda^{2}\right)$. Inserting this in eq. $\left(m_{I}\right)$ annihilates the l.h.s. of this equation. Thus, the linearized $W$ vanishes for asymptotically flat space-times.

It also follows from eq. (17) and from the proper peeling behaviour of $\Lambda$ (see below) that no restriction is impossed on $Q$ (or $W$ to second order in $\Lambda$ ).

A vanishing $W$ (to first order in $\Lambda$ ) gives a big simplification to (21). We rewrite this equation as

$$
\partial_{1}^{5} \bar{\partial}^{2} \check{\partial}^{2} Z=\mathcal{S}\left(\theta_{i}, \zeta, \bar{\zeta}\right),
$$

where the function $\mathcal{S}$ is considered as a source and is given by

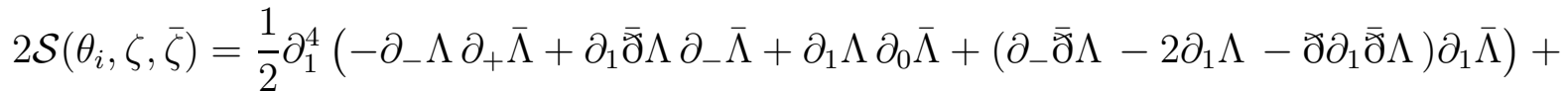

$$
\begin{aligned}
& +\left(\partial_{1} \partial+3 \partial_{-}\right)\left(4 \partial_{+} Q+2 \partial_{1} \bar{\partial} Q\right)+3 \partial_{1}^{2} \Lambda\left(\partial_{1}^{4} \bar{\Lambda}-\partial_{0} \partial_{1}^{3} \bar{\Lambda}-\partial_{+} \partial_{1}^{3} \partial \bar{\Lambda}\right)+\text { c.c. }
\end{aligned}
$$

In order to determine the global null surfaces of asymptotically flat, radiative space-times we integrate eq. (22) using asymptotic boundary conditions on $\Lambda$.

We claim that the final equation reads:

$$
\begin{aligned}
\bar{\delta}^{2} ð^{2} Z= & \left(\varlimsup^{2} \bar{\sigma}_{B}+2 ð Z ð \dot{\bar{\sigma}}_{B}+(ð Z)^{2} \ddot{\bar{\sigma}}_{B}+\sigma_{B} \dot{\bar{\sigma}}_{B}+\text { c.c. }\right)-\int_{-\infty}^{Z} \dot{\sigma}_{B} \dot{\bar{\sigma}}_{B} \mathrm{~d} \mu \\
& -\int_{R}^{\infty} \int_{R_{4}}^{\infty} \mathcal{S}\left(\theta_{i}, \zeta, \bar{\zeta}\right) \mathrm{d} R_{1} \ldots \mathrm{d} R_{5},
\end{aligned}
$$

with $\sigma_{B}$ the free Bondi data at $\mathcal{I}$.

Proof: Our goal is to show that $\lim _{R \rightarrow \infty} \bar{\partial}^{2} \Lambda$ is finite and it is constructed from the free Bondi data $\sigma_{B}$. It would then follow from the peeling conditions that $\lim _{R \rightarrow \infty} \partial_{1}^{i} \overline{\mathrm{\partial}}^{2} \Lambda=0$, for $i=1 \ldots 4$., thus giving only one "constant" of integration to eq. (22).

We consider at $\mathcal{I}$ the Bondi coordinates $(u, \zeta, \bar{\zeta})$ and the l.c. cut given by $u=Z\left(x^{a}, \zeta, \bar{\zeta}\right)$, where $x^{a}$ is an interior point of the space-time.

Bondi coordinates come equipped with a null tetrad $\left(\hat{n}^{a}, \hat{l}^{a}, \hat{m}^{a}, \hat{\bar{m}}^{a}\right)$ that at $\mathcal{I}$ have the following properties: $\hat{n}^{a}$ is a generator of $\mathcal{I}, \hat{m}^{a}$ and $\hat{\bar{m}}^{a}$ are tangent vectors along the Bondi cut $u=$ const, and $\hat{l}^{a}$ points along the direction of the null hypersurface that define the Bondi cuts at $\mathcal{I}$. Furthermore, the vector $\hat{l}^{a}$ is used to construct the shear associated with 
the bondi cuts as $\sigma_{B} \equiv \hat{m}^{a} \hat{\bar{m}}^{b} \nabla_{a} \hat{l}_{b}$, i.e., it contains information about the interior of the space-time.

The l.c. cut $u=Z\left(x^{a}, \zeta, \bar{\zeta}\right)$, on the other hand, is constructed from the intersection of the future null cone from $x^{a}$ with $\mathcal{I}$. This cut also comes equipped with an associated null tetrad $\left(n^{a}, l^{a}, m^{a}, \bar{m}^{a}\right)$ with the following geometrical properties: $n^{a}$ is chosen so that it becomes a generator of $\mathcal{I}, m^{a}$ and $\hat{\bar{m}}^{a}$ so that they are tangent to $\mathcal{I}$. The vector $l^{a}$ points along the generators of the null cone from $x^{a}$. At $\mathcal{I}$ it yields the shear associated with the l.c. cut, namely $\sigma_{Z} \equiv m^{a} \bar{m}^{b} \nabla_{a} l_{b}$.

For every point on the l.c. cut we can write its associated null tetrad in terms of the Bondi tetrad as

$$
\begin{aligned}
& n^{a}=\hat{n}^{a}, \\
& m^{a}=\hat{m}^{a}+ð Z \hat{n}^{a}, \\
& l^{a}=\hat{l}^{a}+\bar{\jmath} Z \hat{m}^{a}+ð Z \hat{\bar{m}}^{a}+\bar{\jmath} Z ð Z \hat{n}^{a} .
\end{aligned}
$$

Defining $\Psi_{2}$ on the null cone from $x^{a}$ as

$$
\Psi_{2}=-\frac{1}{2}\left(C_{a b c d} l^{a} n^{b} l^{c} n^{d}-C_{a b c d} l^{a} n^{b} m^{c} \bar{m}^{d}\right),
$$

and using the relationship between the null tetrads given by (24), we have at $\mathcal{I}$

$$
\Psi_{2}=\Psi_{2 B}-2 ð Z ð \dot{\bar{\sigma}}_{B}-(ð Z)^{2} \ddot{\bar{\sigma}}_{B}
$$

where the dot denotes $\hat{n}^{a} \nabla_{a} \equiv \partial_{u}$.

Solving the Bianchi identity corresponding to $\dot{\Psi}_{2 B}$, i.e. in the Bondi coordinates, we have

$$
\Psi_{2 B}=-\check{\partial}^{2} \bar{\sigma}_{B}-\sigma_{B} \dot{\bar{\sigma}}_{B}+\int_{-\infty}^{u} \dot{\sigma}_{B} \dot{\bar{\sigma}}_{B} \mathrm{~d} \mu
$$

where $\partial$ denote the partial covariant derivative with respect to $\zeta$. Inserting this in equation (26) gives

$$
\Psi_{2}=-ð^{2} \bar{\sigma}_{B}-\sigma_{B} \dot{\bar{\sigma}}_{B}-2 ð Z ð \dot{\bar{\sigma}}_{B}-(ð Z)^{2} \ddot{\bar{\sigma}}_{B}+\int_{-\infty}^{Z} \dot{\sigma}_{B} \dot{\bar{\sigma}}_{B} \mathrm{~d} \mu
$$

On the other hand, Sach's Theorem gives

$$
\overline{\widehat{\partial}}^{2} \check{\partial}^{2} Z=\overline{\widehat{\partial}}_{T}^{2} \sigma_{B}-\overline{\widehat{\jmath}}^{2} \sigma_{Z}
$$

with $\overline{\mathrm{d}}_{T}$ the total derivative, given by

$$
\begin{aligned}
& \overline{\mathrm{\partial}}_{T}^{2} \sigma_{B}(Z, \zeta, \bar{\zeta})=\left(\overline{\grave{\partial}}+\overline{\mathrm{\partial}} Z \partial_{u}\right)\left(\overline{\mathrm{\partial}}+\overline{\mathrm{\partial}} Z \partial_{u}\right) \sigma_{B}, \\
& =\overline{\mathrm{\partial}}^{2} \sigma_{B}+\overline{\mathrm{\partial}}^{2} Z \dot{\sigma}_{B}+2 \overline{\mathrm{\partial}} Z \overline{\mathrm{\partial}} \dot{\sigma}_{B}+(\overline{\mathrm{\partial}} Z)^{2} \ddot{\sigma}_{B}
\end{aligned}
$$

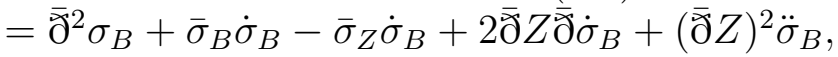

where Sach's Theorem has been used on the last equality. Inserting (29) back on (28) and rearranging terms gives

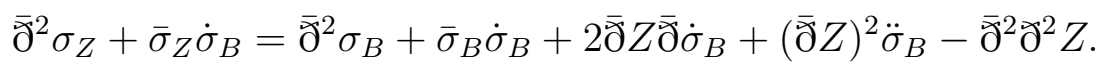


Consider now the following combination

$$
-\Psi_{2}+\bar{\partial}^{2} \sigma_{Z}+\bar{\sigma}_{Z} \dot{\sigma}_{B}
$$

using (27) and (30) gives

$$
\begin{aligned}
\overline{\mathrm{\partial}}^{2} \check{\partial}^{2} Z-\Psi_{2}+\overline{\bar{\partial}}^{2} \sigma_{Z}+\sigma_{Z} \dot{\bar{\sigma}}_{B}= & \left(\check{\partial}^{2} \bar{\sigma}_{B}+\sigma_{B} \dot{\bar{\sigma}}_{B}+2 ð Z \text { ळ } \dot{\bar{\sigma}}_{B}+(ð Z)^{2} \ddot{\bar{\sigma}}_{B}+\text { c.c. }\right)+ \\
& -\int_{-\infty}^{Z} \dot{\sigma}_{B} \dot{\bar{\sigma}}_{B} \mathrm{~d} \mu .
\end{aligned}
$$

The terms on the r.h.s. of (31) are the boundary terms of eq. (22), i.e., they vanish when applying $\partial_{1}$ on this equation. This completes the proof.

\section{THE METRICITY CONDITIONS}

In this section we explore possible generalizations of the l.c.cut equations. The idea is to keep the kinematical arena provided by $\mathcal{I}$ and replace (23) with a simpler equation for the 2-surfaces.

The main motivation for such a generalization is to obtain a field equation that is a good approximation of the conformal Einstein equations. A related motivation is to be able to obtain analytic solutions and a complete knowledge of the solution space.

It is clear that the surfaces satisfying the new equations would not yield conformal vacuum metrics but if they, on the other hand, satisfy $\left(m_{I I}\right)$ they would be characteristic surfaces of an underlying metric of the space-time with a given dynamical evolution due to an effective stress-energy tensor. In particular, we examine two field equations for cuts, one of them appearing in the literature and the other one provided by (23) with $\mathcal{S}=0$.

Before considering the possible generalizations of (23) we analyse the linearized version of this equation, namely,

$$
\check{\partial}^{2} \bar{\partial}^{2} Z^{(1)}\left(x^{a}, \zeta, \bar{\zeta}\right)=\check{\partial}^{2} \bar{\sigma}\left(x^{a} l_{a}, \zeta, \bar{\zeta}\right)+\bar{\varpi}^{2} \sigma\left(x^{a} l_{a}, \zeta, \bar{\zeta}\right)
$$

with $x^{a} l_{a}$ a cut for Minkowski space.

The regular solution to this equation can be written as

$$
Z^{(1)}\left(x^{a}, \zeta, \bar{\zeta}\right)=\int_{S^{2}}\left(\bar{\partial}^{\prime 2} G_{00^{\prime}}\left(\zeta, \zeta^{\prime}\right) \sigma\left(x^{a}, \zeta^{\prime}, \bar{\zeta}^{\prime}\right)+\bar{\partial}^{\prime 2} G_{00^{\prime}}\left(\zeta, \zeta^{\prime}\right) \bar{\sigma}\left(x^{a}, \zeta^{\prime}, \bar{\zeta}^{\prime}\right)\right) \mathrm{d} S^{\prime 2}
$$

where the $G_{00^{\prime}}\left(\zeta, \zeta^{\prime}\right)$ is the corresponding Green function on the sphere and the "prime" implies dependence in the integrations variables $\left(\zeta^{\prime}, \bar{\zeta}^{\prime}\right)$. Applying $\not^{2}$ to the last equation we obtain the following expression for $\Lambda$.

$$
\Lambda\left(x^{a}, \zeta, \bar{\zeta}\right)=\sigma\left(x^{a} l_{a}, \zeta, \bar{\zeta}\right)+\int_{S^{2}} \partial^{2} G_{02^{\prime}} \bar{\sigma}^{\prime} \mathrm{d} S^{\prime 2}
$$

where $G_{02^{\prime}} \equiv \mathrm{\partial}^{\prime 2} G_{00^{\prime}}$.

The linearized metricity condition $\left(m_{I I}\right)$ reads 


$$
\left(m_{I I}\right) \quad \partial_{-} \Lambda-\frac{1}{2} \partial \partial_{1} \Lambda=0 .
$$

Thus, inserting equation (33) in $\left(m_{I I}\right)$ yields after some manipulations

$$
\partial_{-} \Lambda-\frac{1}{2} \partial \partial_{1} \Lambda=-\frac{1}{2} \int_{S^{2}} \dot{\bar{\sigma}}^{\prime}\left(3 ð^{2} G_{02^{\prime}} m^{a} l_{a}^{\prime}+ð^{3} G_{02^{\prime}} l^{a} l_{a}^{\prime}\right) \mathrm{d} S^{\prime 2}=0 .
$$

We have thus shown that the solution to the linearized field equations (32) are the null surfaces of metric $g_{a b}\left(x^{a}\right)$ with coefficients given by (3). Although not needed for the present discussion, we can also show that $W$ vanishes to first order. A straightforward calculation gives

$$
W=\partial_{+} \Lambda+\frac{1}{2} \overline{\bar{\partial}} \partial_{1} \Lambda=-\frac{1}{2} \int_{S^{2}} \dot{\bar{\sigma}}^{\prime}\left(\overline{\mathrm{\partial}}^{2} G_{02^{\prime}} \bar{m}^{a} l_{a}^{\prime}-\overline{\bar{\partial}} \bar{\partial}^{2} G_{02^{\prime}} l^{a} l_{a}^{\prime}\right) \mathrm{d} S^{\prime 2}=0 .
$$

Thus, the solutions to (32) automatically satisfy the metricity conditions $\left(m_{I}\right)$ and $\left(m_{I I}\right)$ to first order.

We now consider possible generalizations of (23) that agree with (32) to first order.

In a recent paper [8], L. Mason conjectured that the following equation,

$$
\varlimsup^{2} \bar{\partial}^{2} Z=\varlimsup^{2} \bar{\sigma}(Z, \zeta, \bar{\zeta})+\bar{\jmath}^{2} \sigma(Z, \zeta, \bar{\zeta}),
$$

should lead to a generalization of the Bach equation for the l.c. cuts. Mason also pointed out that the solutions to this equation could yield a metric of conformal Finsler type, i.e., would not be a conformal metric for the space-time. Since (35) agrees with the linearized version of (23) we should test if $\left(m_{I I}\right)$ is satisfied to second order. We thus consider

$$
\check{\partial}^{2} \bar{\partial}^{2} Z^{(2)}\left(x^{a}, \zeta, \bar{\zeta}\right)=\check{\partial}^{2} \bar{\sigma}\left(Z^{(1)}, \zeta, \bar{\zeta}\right)+\bar{\partial}^{2} \sigma\left(Z^{(1)}, \zeta, \bar{\zeta}\right)
$$

which gives

$$
\Lambda\left(x^{a}, \zeta, \bar{\zeta}\right)=\sigma\left(Z^{(1)}, \zeta \bar{\zeta}\right)+\int_{S^{2}} \not^{2} G_{02^{\prime}} \bar{\sigma}\left(Z^{(1)}, \zeta^{\prime}, \bar{\zeta}^{\prime}\right) \mathrm{d} S^{\prime 2}
$$

Since the second order metricity condition $\left(m_{I I}\right)$ for asymptotically flat spacetimes is identical to (34), we insert the expression for $\Lambda$ in this equation obtaining

$$
\begin{aligned}
\partial_{-} \Lambda-\frac{1}{2} \partial \partial_{1} \Lambda & =-\frac{1}{2} \iint_{S^{2}} \mathrm{~d} S^{\prime 2} \mathrm{~d} S^{\prime \prime 2} \dot{\bar{\sigma}}^{\prime} \frac{\left(l^{[a} m^{b]} l_{[a}^{\prime} m_{b]}^{\prime}\right)^{2}}{\left(l^{c} l_{c}^{\prime}\right)^{4}} l^{[a} m^{b]} l_{[a}^{\prime} l_{b]}^{\prime \prime}\left(G_{02^{\prime \prime}} \sigma^{\prime \prime}+G_{0-2^{\prime \prime}} \bar{\sigma}^{\prime \prime}\right) \\
& \neq 0 .
\end{aligned}
$$

where we have used the explicit form of the Green function and $Z^{(1)}$ to obtain the above expression.

Thus, the solutions to (35) are not characteristic surfaces of an underlying conformal metric. Note that the free data of (35) is given on the cut and thus, the solutions of this equation satisfy Huygens' principle. On the other hand the solutions of (23) are manifestly non-Huygens. It was thought that replacing the r.h.s. of (35) with the free data obtained in the previous section, namely, 


$$
\left(\varlimsup^{2} \bar{\sigma}_{B}+\sigma_{B} \dot{\bar{\sigma}}_{B}+2 ð Z ð \dot{\bar{\sigma}}_{B}+(ð Z)^{2} \ddot{\bar{\sigma}}_{B}+\text { c.c. }\right)-\int_{-\infty}^{Z} \dot{\sigma}_{B} \dot{\bar{\sigma}}_{B} \mathrm{~d} \mu .
$$

could improve the situation. An explicit calculation shows that doing this replacement also fails to satisfy (34).

In both cases the solutions to the field equations would not be null surfaces of any metric since equation $\left(m_{I I}\right)$ is not satisfied. It is worth remarking that although the metricity condition $\left(m_{I I}\right)$ is kinematical in nature it becomes a useful tool to check if solutions to generalizations for the l.c. cut equations are also characteristic surfaces.

\section{CONCLUSIONS}

We have shown that the dynamics of the conformal structure can be written as a single equation for the function $Z$. We proved that this equation is equivalent to the vanishing of the Bach tensor and wrote its explicit form to second order in a perturbation expansion. Using appropriate asymptotic boundary conditions, we obtained the field equations that determine the global null surfaces for asymptotically flat space-times. However, it follows from (E) that, once $Z$ is given, the conformal factor can be explicitly written as a functional of $Z$. Thus, the field equations for $Z$ determine not only the conformal structure but the full geometry of the Einstein spaces. We have also shown that metricity condition $\left(m_{I I}\right)$ becomes an important tool to decide if generalizations of the field equations of the l.c. cuts also yield a conformal structure on the manifold.

One of the main applications of the field equations for the l.c. cuts could be to test the formation of singularities on radiative space-times. Assume one gives regular initial data $\sigma_{B}$ at $\mathcal{I}^{-}$representing incoming gravitational waves. A very important question, for which there is no answer yet, is to determine whether or not singularities will be developed in the future. It is generally accepted that if the data is "strong enough" the self interaction of the gravitational waves will develop a singularity. It is also accepted that if the data is weak, the waves will scatter into the future without forming a singularity. The l.c. cuts, on the other hand, can be used as a tool to detect singularities since their index number is equal to 1 (topological spheres) if the space-time is regular and 0 (open surfaces) if it has a singularity [10]. Thus, if we could show that for a given data $\sigma_{B}$ the solution space of (23) contains a subspace with vanishing index number, we would prove that the space-time has developed a singularity. Note also that the conformal factor $\Omega$ plays no role in this discussion.

Another possible line of research would be to obtain generalizations of the l.c. cut equations that satisfy the metricity conditions or, as pointed out by Mason, to see if the generalized equations are good approximations to general relativity for weak fields.

Future work will address the above issues.

\section{Acknowledgments:}

This research has been partially supported by CONICET, CONICOR and STINT. 


\section{APPENDIX A: PROOF OF PROPOSITION IV.1}

Proof: Using commutators to first order in $\Lambda$, i. e. $f_{i}=\partial_{i} \bar{\delta} \Lambda$. and applying $\left[\partial_{1}^{2}, \widehat{\partial}\right]$ on $\Omega$ gives,

$$
\begin{aligned}
{\left[\partial_{1}^{2}, ð\right] \Omega } & =\partial_{1}^{2} ð \Omega-ð \partial_{1}^{2} \Omega \\
& =\frac{1}{2} \partial_{1}^{2}(W \Omega) .
\end{aligned}
$$

where $\left(\mathrm{E}^{\prime}\right)$ and $\left(m_{I}\right)$ have been used in the last equality. On the other hand, using (8) we compute

$$
\begin{aligned}
{\left[\partial_{1}^{2}, ð\right] } & =\partial_{1}\left[\partial_{1}, \check{\partial}\right]+\left[\partial_{1}, \check{\partial}\right] \partial_{1} \\
& =2 \delta_{1} \partial_{1}+\eta_{1} .
\end{aligned}
$$

Therefore (A1) and (A2), and the first commutation relation given in (6), yield

$$
\partial_{-} \partial_{1} \Omega+\partial_{1} \Lambda \partial_{1} \partial_{+} \Omega=\frac{1}{4} \partial_{1}^{2}(W \Omega)-\frac{1}{2} \eta_{1} \Omega
$$

In a similar way, we obtain the complex conjugate equation by applying $\left[\partial_{1}^{2}, \bar{\partial}\right]$ on $\Omega$. From these two equations we conclude that $\partial_{ \pm} \partial_{1} \Omega=\mathcal{O}(\Lambda)$, thus at first order in $\Lambda$, we can write

$$
\begin{aligned}
& \partial_{-} \partial_{1} \Omega=\frac{1}{4} \partial_{1}^{2}(W \Omega)-\frac{1}{2} \eta_{1} \Omega, \\
& \partial_{+} \partial_{1} \Omega=\frac{1}{4} \partial_{1}^{2}(\bar{W} \Omega)-\frac{1}{2} \bar{\eta}_{1} \Omega .
\end{aligned}
$$

Since these two equations are new differential equations for $\Omega$, they have to be incorporated to the system (18). Therefore we have to find the integrability conditions for this larger system. In order to do this we calculate commutators identities similar to (A2), namely

$$
\delta_{i} \partial_{j}+\delta_{j} \partial_{j}=\partial_{i} \partial_{j} \partial-\left[\partial_{i}, \delta_{j}\right]-ð \partial_{i} \partial_{j}
$$

We now apply the commutators $\left[\partial_{-} \partial_{1}, \widetilde{\partial}\right],\left[\partial_{+} \partial_{1}, \widetilde{\partial}\right]$ and their complex conjugate on $\Omega$. Identity (A4), and equations $\left(m_{I}\right)$ and $\left(\bar{m}_{I}\right)$ allows us to calculate

$$
\begin{aligned}
& \partial_{-}^{2} \Omega+\partial_{1} \Lambda \partial_{-} \partial_{+} \Omega=\frac{1}{2} \partial_{1} \partial_{-}(W \Omega)-\left[\partial_{1}, \delta_{-}\right] \Omega-\partial \partial_{-} \partial_{1} \Omega, \\
& \partial_{0} \partial_{1} \Omega+\partial_{+} \partial_{-} \Omega=\frac{1}{2} \partial_{1} \partial_{+}(W \Omega)-\left[\partial_{1}, \delta_{+}\right] \Omega-\succsim \partial_{+} \partial_{1} \Omega, \\
& \partial_{+}^{2} \Omega+\partial_{1} \bar{\Lambda} \partial_{-} \partial_{+} \Omega=\frac{1}{2} \partial_{1} \partial_{+}(\bar{W} \Omega)-\left[\partial_{1}, \bar{\delta}_{+}\right] \Omega-\bar{\partial} \partial_{+} \partial_{1} \Omega, \\
& \partial_{0} \partial_{1} \Omega+\partial_{+} \partial_{-} \Omega=\frac{1}{2} \partial_{1} \partial_{-}(\bar{W} \Omega)-\left[\partial_{1}, \bar{\delta}_{-}\right] \Omega-\bar{\partial} \partial_{-} \partial_{1} \Omega .
\end{aligned}
$$

Note that using the definition of $W$, one can show that the second and the fourth equations are the same, hence the commutators $\left[\partial_{i} \partial_{j}, \check{\partial}\right]$ and $\left[\partial_{i} \partial_{j}, \overline{\widetilde{\partial}}\right]$ shall give us less than twenty algebraic linear independent equations with respect to the variables $\partial_{i} \partial_{j} \Omega$. 
Once again eq. (A5) are new differential equations for $\Omega$ that have to be incorporated to our system. Therefore in order to obtain the integrability conditions of the system (18) we shall apply on $\Omega$ the commutators $\left[\partial_{i} \partial_{j}, \widetilde{\partial}\right],\left[\partial_{i} \partial_{j}, \bar{\partial}\right]$ and $\left[\partial_{i} \partial_{j}, \partial_{k}\right]$, for $i, j, k=0,+,-, 1$.

By inspection, we conclude that if $\left(m_{I}\right)$ and $\left(m_{I I}\right)$ are satisfied, the system of equations originated by the commutators $\left[\partial_{i} \partial_{j}, \check{\partial}\right]$ and $\left[\partial_{i} \partial_{j}, \overline{\widetilde{\partial}}\right]$ yield eight linear independent differential equations for $\Omega$, namely

$$
\begin{aligned}
& \partial_{-} \partial_{1} \Omega=\frac{1}{4} \partial_{1}^{2}(W \Omega)-\frac{1}{2} \eta_{1} \Omega, \\
& \partial_{-}^{2} \Omega+\partial_{1} \Lambda \partial_{-} \partial_{+} \Omega=\frac{1}{2} \partial_{1} \partial_{-}(W \Omega)-\left[\partial_{1}, \delta_{-}\right] \Omega-\partial_{-} \partial_{1} \Omega \\
& \partial_{0} \partial_{1} \Omega+\partial_{+} \partial_{-} \Omega=\frac{1}{2} \partial_{1} \partial_{+}(W \Omega)-\left[\partial_{1}, \delta_{+}\right] \Omega-\partial \partial_{+} \partial_{1} \Omega \\
& \partial_{0} \partial_{+} \Omega+\frac{1}{2} \partial \partial_{1} \bar{\Lambda} \partial_{+} \partial_{-} \Omega=\frac{1}{2}\left(\frac{1}{2} \partial_{+}^{2}(W \Omega)+\partial_{1}^{2}(\bar{W} \Omega)-2 \bar{\eta}_{1} \Omega-\left[\partial_{+}, \delta_{+}\right] \Omega+\right. \\
& \left.-\frac{1}{2} ð \partial_{+} \partial_{1}(\bar{W} \Omega)+ð \bar{\partial}\left[\partial_{1}, \bar{\delta}_{+}\right] \Omega\right)+\frac{1}{2} ð \bar{\partial} \partial_{1} \partial_{+} \Omega \\
& \partial_{0}^{2} \Omega-\left(2-f_{+}-\frac{1}{2} \partial^{2} \partial_{1} \bar{\Lambda}\right) \partial_{0} \partial_{1} \Omega=\frac{1}{2} \partial_{+} \partial_{0}(W \Omega)-\left[\partial_{+}, \delta_{0}\right] \Omega-\frac{1}{2} \partial\left(\frac{1}{2} \partial_{+}^{2}(W \Omega)+\right. \\
& \partial_{1}^{2}(\bar{W} \Omega)-2 \bar{\eta}_{1} \Omega-\left[\partial_{+}, \delta_{+}\right] \Omega-\frac{1}{2} \partial \partial_{+} \partial_{1}(\bar{W} \Omega)+ \\
& \left.+{ }^{\bar{\partial}}\left[\partial_{1}, \bar{\delta}_{+}\right] \Omega\right)-\frac{1}{2} \varlimsup^{2} \bar{\partial} \partial_{1} \partial_{+} \Omega,
\end{aligned}
$$

and their complex conjugate. Note that the third and the last equations are real.

Remark A.1 Observing this system, we conclude that it can be generated by applying $\partial$ and $\overline{\mathrm{\partial}}$ an appropriate number of times on equation ( $\left.E^{\prime}\right)$. Consequently the equations of this system are the components of the trace free part of the field equations calculated in the coordinates frame $\theta_{i}^{a}$. Moreover, note that this system has eight equations and nine unknowns, therefore one of them shall be a parameter, for example $\partial_{0} \partial_{1} \Omega$. This parameter corresponds to the trace of the field equations.

It now remains to consider the commutators $\left[\partial_{i} \partial_{j}, \partial_{k}\right]$. We begin applying $\left[\partial_{1}^{2}, \partial_{-}\right]$on $\Omega$ as follows.

$$
\begin{aligned}
0 \equiv 4\left[\partial_{1}^{2}, \partial_{-}\right] \Omega & =4 \partial_{1}\left(\partial_{1} \partial_{-} \Omega\right)-4 \partial_{-}\left(\partial_{1}^{2} \Omega\right) \\
& =\partial_{1}^{3}(W \Omega)-2 \eta_{2} \Omega
\end{aligned}
$$

where $\eta_{2}=\left[\partial_{1}, \eta_{1}\right]$, and (E') and the first equation of (A6) have been used in the last equality. Similarly, we calculate its complex conjugate expression.

$$
0 \equiv 4\left[\partial_{1}^{2}, \partial_{+}\right] \Omega=\partial_{1}^{3}(\bar{W} \Omega)-2 \bar{\eta}_{2} \Omega .
$$

Note that (A7) and (A8) only contain first derivatives of $\Omega$ since all second derivatives can be replaced using ( $\left.\mathrm{E}^{\prime}\right)$. 
We obtain the next integrability condition by applying the commutator $\left[\partial_{+} \partial_{1}, \partial_{-}\right]$on $\Omega$

$$
\begin{aligned}
0 \equiv 4\left[\partial_{+} \partial_{1}, \partial_{-}\right] \Omega & =4 \partial_{+}\left(\partial_{1} \partial_{-} \Omega\right)-4 \partial_{-}\left(\partial_{+} \partial_{1} \Omega\right) \\
& =\partial_{1}^{2} \partial_{+}(W \Omega)-\partial_{1}^{2} \partial_{-}(\bar{W} \Omega)-2\left(\partial_{+} \eta_{1} \Omega-\partial_{-} \bar{\eta}_{1} \Omega\right),
\end{aligned}
$$

here we have used the expressions for $\partial_{1} \partial_{ \pm} \Omega$ given in the system (A6). Furthermore we calculate

$$
\begin{aligned}
0 \equiv 4\left[\partial_{1}^{2}, \partial_{0}\right] \Omega+4\left[\partial_{1} \partial_{-}, \partial_{+}\right] \Omega & =4 \partial_{1}\left(\partial_{0} \partial_{1} \Omega+\partial_{-} \partial_{+} \Omega\right)-4 \partial_{+}\left(\partial_{1} \partial_{-} \Omega\right) \\
& =\partial_{1}^{2} \partial_{+}(W \Omega)-4 \partial_{1}\left[\partial_{1}, \delta_{+}\right] \Omega-4 \partial_{1} \partial \partial_{+} \partial_{1} \Omega+2 \partial_{+} \eta_{1} \Omega
\end{aligned}
$$

the last equations have been obtained using once again the corresponding expression for the second derivatives $\partial_{0} \partial_{1} \Omega+\partial_{-} \partial_{+} \Omega$ and $\partial_{1} \partial_{-} \Omega$ given in (A6). Finally, replacing $\partial_{1} \partial_{+} \Omega$, using the integrability condition (A9) and commutating a couple of times, we obtain

$$
0 \equiv 4\left[\partial_{1}^{2}, \partial_{0}\right] \Omega+4\left[\partial_{1} \partial_{-}, \partial_{+}\right] \Omega=-ð \partial_{1}^{3}(\bar{W} \Omega)+2 ð \bar{\eta}_{2} \Omega
$$

Observe that the integrability condition ( $\mathrm{A10}$ ) can be obtained equivalently by applying on on equation (A7) thus, as in the case of the field equations, we generate linear combinations of the integrability condition corresponding to $\left[\partial_{i} \partial_{j}, \partial_{k}\right] \Omega$ by applying $\widetilde{\partial}$ and $\bar{\partial}$ an appropriate number of times on equation (A7).

Summarizing, the original system has become even larger and it remains to find equations independent of $\Omega$ as integrability conditions. One of these conditions can be obtained by applying $\partial_{1}$ on equation (A10) and using (8) as follows.

$$
\begin{aligned}
4 \partial_{1} \mathrm{\partial}\left[\partial_{1}^{2}, \partial_{+}\right] \Omega & =\partial_{1} \partial\left(\partial_{1}^{3}(\bar{W} \Omega)-2 \bar{\eta}_{2} \Omega\right) \\
& =\partial_{1}^{4} \mathrm{\partial}(\bar{W} \Omega)-\partial_{1}\left[\partial_{1}^{3}, \check{\partial}\right](\bar{W} \Omega)-2 \partial_{1} \partial \bar{\eta}_{2} \Omega, \\
& =\partial_{1}^{4} \mathrm{\partial}(\bar{W} \Omega)-3 \partial_{-} \partial_{1}^{3}(\bar{W} \Omega)-2 \partial_{1} \partial \bar{\eta}_{2} \Omega, \\
& =\partial_{1}^{4} \partial(\bar{W} \Omega)-3 \partial_{-}\left(4\left[\partial_{1}^{2}, \partial_{+}\right] \Omega+2 \bar{\eta}_{2} \Omega\right)-2 \partial_{1} \mathrm{\partial} \bar{\eta}_{2} \Omega,
\end{aligned}
$$

in the last equation we have used the integrability condition (A8). Consequently we write

$$
\begin{aligned}
& 0 \equiv 4 \partial_{1} \partial\left[\partial_{1}^{2}, \partial_{+}\right] \Omega+12 \partial_{-}\left[\partial_{1}^{2}, \partial_{+}\right] \Omega=\partial_{1}^{4} \partial(\bar{W} \Omega)-6 \partial_{-} \bar{\eta}_{2} \Omega-2 \partial_{1} \partial \bar{\eta}_{2} \Omega \\
& =\Omega \partial_{1}^{4} \mathrm{\partial} \bar{W}+4 \partial_{1}^{3} \mathrm{\partial} \bar{W} \partial_{1} \Omega-6\left[\partial_{-}, \bar{\eta}_{2}\right] \Omega-2\left[\partial_{1} \partial, \bar{\eta}_{2}\right] \Omega,
\end{aligned}
$$

here we have used the system (A6), and equations (E') and $\left(m_{I}\right)$. Note that this equation contains only first derivatives of $\Omega$. Using now the expression for $W$ and calculating the commutators $\left[\partial_{+}, \bar{\eta}_{2}\right]$ and $\left[\partial_{1} \precsim, \bar{\eta}_{2}\right]$, we find that the coefficients enclosed to $\partial_{i} \Omega$ are zero and as consequence the equation (A12) becomes

$$
\partial_{1}^{4} \precsim \bar{W}=0 .
$$

Note that we have a similar equation corresponding to the complex conjugate commutators, i.e $\partial_{1}^{4} \overline{\mathrm{d}} W=0$.

Since at first order $\precsim \bar{\partial} \bar{W}=\overline{\mathrm{\partial}} W$, we get the same integrability condition. Using again the definition of $W$, we obtain a differential equation for $\Lambda$, namely

$$
\partial_{1}^{5} \check{\partial}^{2} \bar{\Lambda}=0
$$

This completes the proof. $\square$ 


\section{APPENDIX B: PROOF OF PROPOSITION IV.2}

Proof: Following the same approach that leads to (A6) but using the full commutators gives the following system of equations

$$
\begin{aligned}
\partial_{-} \partial_{1} \Omega+\partial_{1} \Lambda \partial_{1} \partial_{+} \Omega= & \frac{1}{4} \partial_{1}^{2}(W \Omega)-\frac{1}{2} \eta_{1} \Omega-\frac{1}{2} \partial(Q \Omega)-f_{1} Q \Omega \\
\partial_{-}^{2} \Omega+\partial_{1} \Lambda \partial_{-} \partial_{+} \Omega= & \frac{1}{2} \partial_{1} \partial_{-}(W \Omega)-\left[\delta_{-}, \partial\right] \Omega-f_{-} Q \Omega-\partial_{-} \Lambda \partial_{+} \partial_{1} \Omega+ \\
& -f_{1} \partial_{-} \partial_{1} \Omega-\partial\left(\partial_{1} \partial_{-} \Omega\right), \\
\partial_{0} \partial_{1} \Omega+q \partial_{+} \partial_{-} \Omega= & \frac{1}{2} \partial_{+} \partial_{1}(\Omega W)-\left[\delta_{+}, \partial\right] \Omega-f_{+} Q \Omega-\left(f_{1}+\partial_{+} \Lambda\right) \partial_{1} \partial_{+} \Omega+ \\
& -\partial \partial_{1} \partial_{+} \Omega+\partial_{1} \Lambda\left(\frac{1}{2} \partial_{1} \partial_{-}(W \Omega)-\left[\delta_{-}, \partial\right] \Omega_{+}\right. \\
& \left.-f_{-} Q \Omega-\partial_{-} \Lambda \partial_{+} \partial_{1} \Omega-f_{1} \partial_{-} \partial_{1} \Omega-ð\left(\partial_{1} \partial_{-} \Omega\right)\right)
\end{aligned}
$$

and their complex conjugate. Since the last equation is real, this system contains only five linear independent equations.

The remaining equations for $\partial_{0}^{2} \Omega$ and $\partial_{+} \partial_{0} \Omega$ and its complex conjugate are obtained by applying $\partial$ and $\widetilde{\partial}^{2}$ on the last equation of the system (B1).

We now consider the commutators $\left[\partial_{i} \partial_{j}, \partial_{k}\right] \Omega$. Taking the first equation of (B1) and commuting with $\partial_{1}$ gives

$$
\begin{aligned}
{\left[\partial_{1}^{2}, \partial_{-}\right] \Omega+\partial_{1} \Lambda\left[\partial_{1}^{2}, \partial_{+}\right] \Omega } & =\left[\partial_{1}^{2}, \delta_{1}\right] \Omega-f_{1}\left[\partial_{1}^{2}, \partial_{1}\right] \Omega-2 \eta_{1} \partial_{1} \Omega-\eta_{2} \Omega \\
& =\partial_{1}\left(\partial_{1} \delta_{1} \Omega\right)-\delta_{1}(Q \Omega)-2 \eta_{1} \partial_{1} \Omega-\eta_{2} \Omega \\
& =\frac{1}{4} \partial_{1}^{3}(W \Omega)-\frac{1}{2} \partial_{1} \partial(Q \Omega)-\frac{3}{2} \eta_{1} \partial_{1} \Omega-\frac{1}{2} \eta_{2} \Omega-\delta_{1}(Q \Omega),
\end{aligned}
$$

Thus, algebraically solving for (B2) and its c.c. yields

$$
0 \equiv 4\left[\partial_{1}^{2}, \partial_{+}\right] \Omega=c_{11+}
$$

where

$$
\begin{aligned}
c_{11+} \equiv & \frac{1}{q}\left(\partial_{1}^{3}(\bar{W} \Omega)-\partial_{1} \bar{\Lambda} \partial_{1}^{3}(W \Omega)\right)-\frac{6}{q}\left(\bar{\eta}_{1} \partial_{1} \Omega-\partial_{1} \bar{\Lambda} \eta_{1} \partial_{1} \Omega\right)+ \\
& -\frac{2}{q}\left(\bar{\eta}_{2} \Omega-\partial_{1} \bar{\Lambda} \eta_{2} \Omega\right)-\frac{2}{q}\left(\partial_{1} \overline{\widehat{\partial}}(Q \Omega)-\partial_{1} \bar{\Lambda} \partial_{1} \partial(Q \Omega)\right) \\
& -\frac{2}{q}\left(\bar{\delta}_{1}(Q \Omega)-\partial_{1} \bar{\Lambda} \delta_{1}(Q \Omega)\right) .
\end{aligned}
$$

As in the first order calculation we obtain new integrability conditions calculating $\left[\partial_{1}^{2}, \partial_{+}\right] \Omega$ and $\left[\partial_{1} \partial_{+}, \partial_{-}\right] \Omega$.

In order to obtain the integrability condition corresponding to equation (A10), we follow the same procedure as in the first order calculation giving 


$$
\begin{aligned}
0 & =4\left[\partial_{1}^{2}, \partial_{0}\right] \Omega+4 q\left[\partial_{1} \partial_{-}, \partial_{+}\right] \Omega \\
& =4\left(\partial_{1}\left(\partial_{0} \partial_{1} \Omega+q \partial_{+} \partial_{-} \Omega\right)-\partial_{0}\left(\partial_{1}^{2} \Omega\right)-q \partial_{+}\left(\partial_{1} \partial_{-} \Omega\right)-\partial_{1} q\left(\partial_{-} \partial_{+} \Omega\right)\right) .
\end{aligned}
$$

Using the corresponding equations of the system (B1), and (E), and the integrability condition given by $\left[\partial_{1} \partial_{+}, \partial_{-}\right] \Omega=0$, we obtain

$$
\begin{aligned}
0 & \equiv 4\left[\partial_{1}, \partial_{0}\right] \Omega+4 q\left[\partial_{1} \partial_{-}, \partial_{+}\right] \Omega \\
& =-\left(\partial c_{11+}+F c_{11+}\right),
\end{aligned}
$$

where

$$
F=2 f_{1}+\partial_{+} \Lambda-\frac{1}{2}\left(\overline{\widehat{\partial}} \partial_{1} \Lambda+\bar{W} \partial_{1} \Lambda\right) .
$$

Note that equation (B5) can be obtained by applying the operator $(\widetilde{\partial}+F)$ on the integrability condition (B3).

The integrability condition independent of $\partial_{i} \Omega$ corresponding to equation (A12) can essentially be calculated following the same procedure as in the first order calculation.

Namely, we take $\partial_{1}$ to equation $(\mathbb{B} 5)$ and commutate $\partial_{1}^{3}$ with $\precsim$. On the other hand we apply the operator $\left(\partial_{-}+\frac{1}{2}\left(\overline{\bar{\partial}} \partial_{1} \Lambda+\bar{W} \partial_{1} \Lambda\right) \partial_{1}+\partial_{1} \Lambda \partial_{+}\right)$on equation (B3) and replace this result in the previous equation or equivalently

$$
\begin{aligned}
& 0 \equiv 4 \partial_{1}\left(\check{\partial}\left[\partial_{1}^{2}, \partial_{+}\right] \Omega+F\left[\partial_{1}^{2}, \partial_{+}\right] \Omega\right)+12\left(\partial_{-}+\frac{1}{2}\left(\overline{\widetilde{\partial}} \partial_{1} \Lambda+\bar{W} \partial_{1} \Lambda\right) \partial_{1}+\partial_{1} \Lambda \partial_{+}\right)\left[\partial_{1}^{2}, \partial_{+}\right] \Omega \\
& =\partial_{1}\left(ð c_{11+}+F c_{11+}\right)+3\left(\partial_{-}+\frac{1}{2}\left(\bar{\partial} \partial_{1} \Lambda+\bar{W} \partial_{1} \Lambda\right) \partial_{1}+\partial_{1} \Lambda \partial_{+}\right) c_{11+} \cdot
\end{aligned}
$$

When this expression is written explicitly using the previous equations, the r.h.s. of this integrability condition has no second derivatives of $\Omega$ except for a term with $\partial_{0} \partial_{1} \Omega$. The coefficient of this term is purely imaginary, thus the integrability condition is given by the real part of (B7).

We also claim that the real part of eq. (B7) does not contain first derivatives of $\Omega$. To prove this, instead of doing a straightforward calculation, we follow a tensorial approach establishing a correspondence between the integrability condition (B7) and the coordinates components of the Bach tensor (for details see [9]). 
[1] E.T. Newman and R. Penrose, J. Math. Phys. 7, 863 (1966).

[2] C.N. Kozameh and E. T. Newman. Topological Properties and Global of Space-Time, edited by A. Held by P. Bergmann and V. de Sabbata, Plenum,New York (1986).

[3] C.N. Kozameh and E. T. Newman. Theory of light cone cuts of null infinity. J. Math. Phys. 24, 2481-2489 (1983).

[4] Simonetta Fritelli, Ezra T. Newman and Carlos Kozameh.GR via characteristic surfaces, J. Math. Phys. 36, 4986-5004 (1995).

[5] Simonetta Fritelli, Ezra T. Newman and Carlos Kozameh.On the Dynamics of Characteristic Surfaces, J. Math. Phys. 36, 6397-6416 (1995).

[6] R. Bach, Math. Zeitschrift 9, 110 (1921).

[7] C. N. Kozameh, E. T. Newman and K. P. Tod, Conformal Einstein Spaces, Gen. Rel. Grav. 17, 4 (1985).

[8] L. J. Mason. The vacuum and Bach equations in terms of light cone cuts, J. Math. Phys. 36, 3704-3721 (1995).

[9] A.T. Rojas, Global Formulation of GR, Ph.D. Thesis, in preparation (1997).

[10] C. Kozameh, P. Lamberti and O. Reula, Global Aspects of Light Cone Cuts, J. Math. Phys. 32, 3423-3426 (1991). 